\title{
Attribute Embedding with Visual-Semantic Ambiguity Removal for Zero-shot Learning
}

\author{
Yang Long ${ }^{1}$ \\ ylong2@sheffield.ac.uk \\ Li Liu ${ }^{2}$ \\ li2.liu@northumbria.ac.uk \\ Ling Shao ${ }^{2}$ \\ ling.shao@ieee.org
}
${ }^{1}$ Department of Electronic and Electrical Engineering The University of Sheffield Sheffield, UK

${ }^{2}$ Department of Computer and Information Sciences Northumbria University Newcastle upon Tyne, UK
Conventional zero-shot learning (ZSL) methods recognise an unseen instance by projecting its visual features to a semantic space that is shared by both seen and unseen categories [1, 2]. However, we observe that such a one-way paradigm suffers from the visual-semantic ambiguity problem. As shown in Fig. 1, the semantic concepts (e.g. attributes or classes) cannot explicitly correspond to visual patterns, and similar visual features may come from different classes. Such a problem can lead to a huge variance in the visual features for each attribute.

In this paper, we propose the VisualSemantic Ambiguity Removal (VSAR) algorithm to address such a problem. In particular, we propose a novel latent attribute space $\mathcal{V}$ to mitigate the gap between visual and semantic spaces $\mathcal{X}$ and $\mathcal{A}$ :

$$
J=\left\|\mathcal{X}-U_{1} \mathcal{V}\right\|_{F}^{2}+\alpha\left\|\mathcal{A}-U_{2} \mathcal{V}\right\|_{F}^{2}+\lambda \mathcal{R},
$$

where $U_{1}$ and $U_{2}$ are two projection matrices. $\mathcal{R}$ is a Dual-graph regularisation that combines two supervised graphs $W_{\mathcal{X}}$ and $W_{\mathcal{A}}$ that model the intrinsic data structures in $\mathcal{X}$ and $\mathcal{A}$. In the embedding space $\mathcal{V}$, we expect that if the vertices in both graphs are connected, each pair of embedded points $v_{i}$ and $v_{j}$ are also closed to each other. However, for the visual-semantic ambiguity problem, $W_{\mathcal{X}}$ and $W_{\mathcal{A}}$ usually give contradictory results. To compromise such conflict, we linearly combine the two graphs, i.e. $W_{i j}=W_{\mathcal{X}_{i j}}+\alpha W_{\mathcal{A}_{i j}}$. The resulted regularisation is:

$$
\mathcal{R}=\frac{1}{2} \sum_{i, j=1}^{N}\left\|v_{i}-v_{j}\right\|^{2} W_{i j}=\operatorname{Tr}\left(\mathcal{V} L \mathcal{V}^{T}\right),
$$

where $D$ is the degree matrix of $W, D_{i i}=\sum_{i} w_{i j}$. $L$ is known as graph Laplacian matrix $L=D-W$

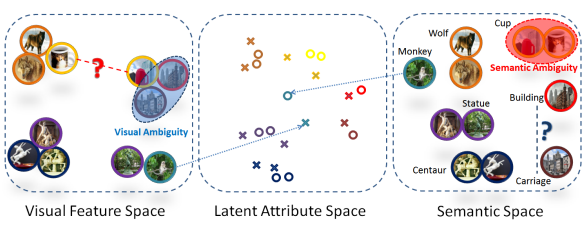

Figure 1: Visual Ambiguity (in blue oval): the image of a carriage is taken with a building background. It cannot recover the semantic distance (blue question mark) to the building category. Semantic Ambiguity (in red oval): the cup printed with a wolf and the cup-like building share the same name which can lead to a large visual variance (the red question mark). After embedding to the latent attribute space using VSAR, such ambiguity is mitigated.

and $\operatorname{Tr}($.$) computes the trace of a matrix.$

Once we obtain the latent attribute embedding $\mathcal{V}$ of the seen data, performing zeroshot recognition is straightforward via leastsquare approximation between $\mathcal{V}$ and $\{\mathcal{A}, \mathcal{X}\}$. During the test, given unseen category names and their attributes in pairs: $\left\{\mathcal{Y}_{u}, \mathcal{A}_{u}\right\}$. We firstly embed all unseen attributes $\mathcal{A}_{u}$ into the latent embedding space as references: $\mathcal{V}_{u}=$ $\mathcal{V} \mathcal{A}^{T}\left(\mathcal{A} \mathcal{A}^{T}\right)^{-1} \mathcal{A}_{u}$. Given a test unseen instance $\hat{x}$, its embedded latent attribute representation is: $\hat{v}=\mathcal{V} \mathcal{X}^{T}\left(\mathcal{X} \mathcal{X}^{T}\right)^{-1} \hat{x}$. Finally, we adopt a simple $\mathrm{NN}$ classifier to predict the category label $\hat{c}$ :

$$
\hat{c}=\arg \min _{c}\left\|\hat{v}-v_{c}\right\|^{2}, \text { where } v_{c} \in \mathcal{V}_{u} .
$$

[1] Zeynep Akata, Florent Perronnin, Zaid Harchaoui, and Cordelia Schmid. Label-embedding for attribute-based classification. In CVPR, 2013.

[2] Christoph H Lampert, Hannes Nickisch, and Stefan Harmeling. Learning to detect unseen object classes by between-class attribute transfer. In CVPR, 2009. 